\title{
First Magnetic Doppler Images of a roAp star
}

\author{
T. Lüftinger, ${ }^{1}$ O. Kochukhov, ${ }^{2}$ T. Ryabchikova, ${ }^{1,3}$ W. W. Weiss, ${ }^{1}$ I. Ilyin ${ }^{4}$ \\ ${ }^{1}$ Institute of Astronomy, University of Vienna, 1180 Vienna, Austria \\ 2 Department of Astronomy and Space Physics, Uppsala University Box 515, SE-751 20 Uppsala, Sweden \\ ${ }^{3}$ Institute of Astronomy, Russian Academy of Sciences, Pyatnitskaya 48, 119017 Moscow, Russia \\ ${ }^{4}$ Astrophysikalisches Institut Potsdam, An der Sternwarte 16, D-14482 Potsdam, Germany
}

\begin{abstract}
We present the first analysis of the magnetic field geometry and elemental abundance distributions on the surface of a rapidly oscillating $A p$ (roAp) star, using an elaborate magnetic Doppler Imaging (MDI) code (Piskunov et al. 2002, Kochukhov et al. 2002), INVERS10, which allows to reconstruct simultaneously and consistently the magnetic field geometry and abundance distributions on a stellar surface without any a priori assumptions. We analysed Stokes $I$ and $V$ time series obtained with the SOFIN polarimeter and recovered the magnetic field and surface abundance structures of $\mathrm{Fe}$ and $\mathrm{Nd}$ (among others). These two elements are found to be anticorrelated.
\end{abstract}

\section{Introduction and MDI analysis}

Still very little is known about the origin and structure of magnetic fields and their connection and interaction with surface abundance patches, pulsation, and stratification. Ap stars exhibit magnetic fields that appear to be highly ordered, very stable, and often very strong. Many Ap stars also show dramatic line profile variations synchronized to stellar rotation, which is attributed to oblique magnetic and pulsation axes and to the presence of a non-uniform distribution of chemical elements on their surface. An important subgroup of the Ap stars, the rapidly oscillating $A p(r o A p)$ stars, in addition, exhibit high-overtone, low-degree, non-radial $p$-mode pulsations with periods of 6-21 minutes. HD 24712 (HR 1217, DO Eri) is the best studied roAp star that was discovered to be a pulsator by Kurtz (1982). Matthews et al. (1988) found radial velocity variations with an amplitude of $0.4 \pm 0.05 \mathrm{~km} \mathrm{~s}^{-1}$ at the main photometric period of $6.14 \mathrm{~min}$. We found atmospheric parameters of $T_{\text {eff }}=7350 \mathrm{~K}$ and $\log g=4.2$, and derived $v \sin i=5.6 \mathrm{~km} \mathrm{~s}^{-1}$.

Spectropolarimetric observations of HD 24712 were carried out in October and November 2003, using the high resolution échelle spectrograph SOFIN, attached to Nordic Optical Telescope (NOT), La Palma, Spain, with a nominal resolving power of $\approx 80000$. Rotational phases of HD 24712 were calculated according to the ephemeris and rotation period obtained by Ryabchikova et al. (2005): $H J D\left(\left\langle B_{z}\right\rangle_{\max }\right)=2453235.18(40)+12.45877(16) \mathrm{d}$.

The determination of the geometry of the magnetic field on the surface of HD 24712 was performed choosing 7 different Fe I and 5 different Nd III lines suitable for magnetic Doppler imaging. The tilt and the azimuth angle of the stellar rotational axis, $i$ and $\Theta$, were used as determined by Bagnulo et al. (1995): $i=137^{\circ}$ and $\Theta=4^{\circ}$. A clear dipolar geometry (Fig. 1) yielded the best fit to the observed Stokes $I$ and $V$ line profiles in our magnetic Doppler imaging analysis. The resulting magnetic field strength varies between $+2.2 \mathrm{kG}$ and $+4.4 \mathrm{kG}$. The surface abundances of Fe and Nd mapped simultaneously with the magnetic field geometry are presented in Fig. 2. It can be clearly seen that the abundances of both elements are globally structured. Nd is extremely overabundant, varying between -8.0 and -7.0 dex $(-10.59$ dex solar $)$. Both elements seem to be perfectly anticorrelated: $\mathrm{Fe}$ is accumulated where $\mathrm{Nd}$ is depleted, and minimum $\mathrm{Fe}$ abundance can be found where $\mathrm{Nd}$ is 
at its maximum. We find that the Fe abundance enhancement region is associated with the area of minimum magnetic field strength around $\phi \simeq 0.5$, whereas the Nd map shows its area of maximum abundance around the magnetic field maximum, where the positive magnetic pole is orientated towards the observer. The additional 14 chemical elements we mapped, including $\mathrm{Mg}, \mathrm{Ca}, \mathrm{Sc}, \mathrm{Ti}, \mathrm{Cr}, \mathrm{Co}, \mathrm{Ni}, \mathrm{Y}, \mathrm{La}, \mathrm{Ce}, \mathrm{Pr}, \mathrm{Gd}, \mathrm{Tb}$, and $\mathrm{Dy}$, exhibit comparable behaviour. Due to limited space, we are not able to present details for all elements in this paper and would like to refer to a forthcoming publication (Lüftinger et al. 2007).

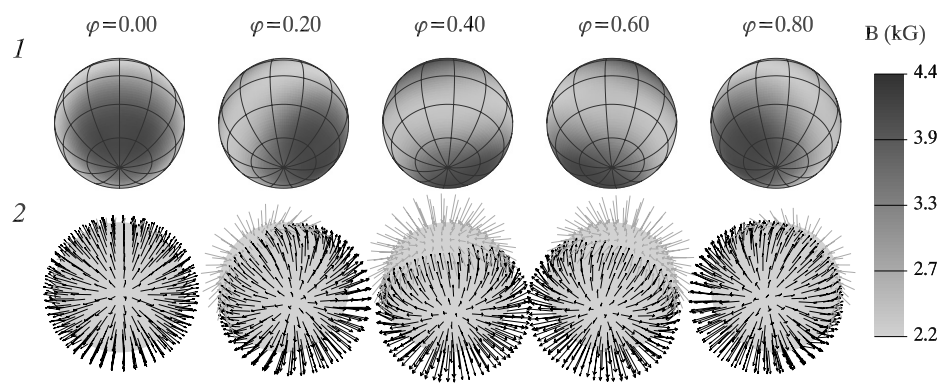

Figure 1: First mapping of the distribution of magnetic field strength (a) and field orientation (b) on the surface of a roAp star. Top: distribution of the field strength, bottom: orientation of the magnetic vectors. The black arrows correspond to field vectors pointing outwards the stellar surface, while grey vectors are pointing inside.

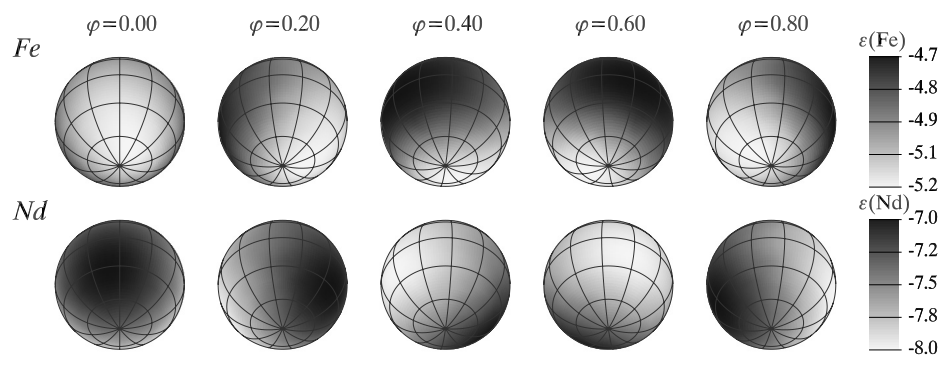

Figure 2: The abundance distributions of Fe and NdIII on the surface of HD 24712. These maps were derived using the Stokes $I$ and $V$ spectra.

Acknowledgments. This work was supported by the Austrian Science Fund (FWFPP17890).

\section{References}

Bagnulo S., Landi Degl'Innocenti E., Landolfi M., Leroy J. L., 1995, A\&A, 295, 459

Kochukhov O., Piskunov N., 2002, A\&A, 388, 868

Kurtz D. W., 1982, MNRAS, 200, 807

Lüftinger T., Kochukhov O., Ryabchikova T., Weiss W. W., Ilyin I., 2007, in Romanyuk I. I., Kudryavtsev D. O., eds, Magnetic Stars. Special Astrophysical Observatory, Russian Academy of Sciences, in press

Matthews J. M., Wehlau W. H., Walker G. A. H., Yang S., 1988, ApJ, 324, 1099

Piskunov N., Kochukhov O., 2002, A\&A, 381, 736 